\title{
Comparison Effect of Direct Trocar and Veress Needle Entry Techniques on Gut Functions after Laparoscopic Procedures: Randomized Clinical Trial
}

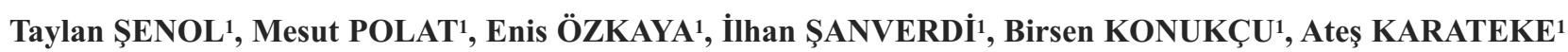 \\ Istanbul, Turkey
}

\begin{abstract}
OBJECTIVE: The aim of this study was to compare the effect of two different laparoscopic entry methods on postoperative gastrointestinal functions.

STUDY DESIGN: A total of 108 women who underwent gynecological operation via laparoscopic approach with different indications were randomly assigned to 2 groups: In Group 1 pneumoperiteneum was achieved by direct trocar entry $(n=72)$, while in group 2, Veress needle was used.

RESULTS: Correlation analyses showed a significant association between the technique for abdominal entry and postoperative hemoglobin and hematocrit concentrations, time to maximal intraabdominal pressure and the body mass index. Comparison of groups with different laparoscopic entry techniques showed a significant difference between groups in terms of postoperative hemoglobin and hematocrit concentrations, time to obtain maximal intraabdominal pressure and time to first flatulence $(p<0.05)$. No intra or postoperative complications was observed.
\end{abstract}

CONCLUSION: Direct or Veress needle entry methods were both safe to create pneumoperitoneum with similar postoperative gastrointestinal functions except for earlier first flatulence in Veress needle group while direct trocar entry was found to be associated with favorable postoperative blood count and shorter duration to obtain enough intraperitoneal pressure.

Keywords: Gastrointestinal motility, Laparoscopic surgical procedure, Peritoneal irrigation, Postoperative complications, Trocar

Gynecol Obstet Reprod Med 2016;22(3):156-159 DOI: 10.21613/GORM.2016.65

\section{Introduction}

Laparoscopic operations have been frequently preferred surgical approaches for the last two decades. Laparoscopic procedures have some advantages over laparotomy including shorter duration of hospitalization, faster recovery so that earlier return to daily life. Laparoscopic surgical manipulations need an empty space provided by the $\mathrm{CO}_{2}$ insufflation into the abdomen at an amount that the patient can tolerate. In recently published studies, there have been some evidences about some structural, metabolic and immune changes on the peritoneum caused by insufflated $\mathrm{CO}_{2}(1,2)$. Also some studies have shown that these changes were due to cooling effect of cold gas insufflation that leads to drying of peritoneal surface free from the type of insufflated gas (2). Furthermore, it has been reported that after an abdominal operation polymorphonuclear

\section{${ }^{1}$ Zeynep Kamil Training and Research Hospital, Obstetric and Gynecology Department, Istanbul}

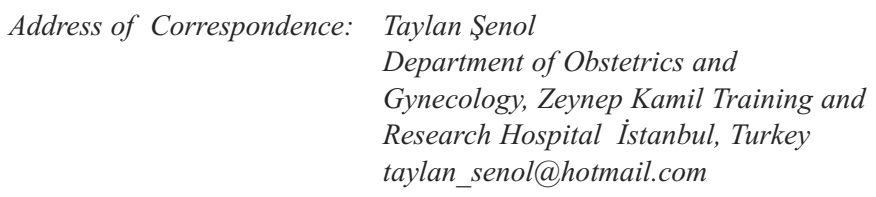

leucocyte flow occurs and macrophage accumulation follows at the site of intervention and finally mast cell degranulation leads to secretion of vasoactive materials that increase vascular permeability (3). Some changes after gas insufflation have been introduced including production of some cytokines by the macrophages and also some decrease in phagocytic function of macrophages $(4,5)$. Also it is well known that diffusion of $\mathrm{CO}_{2}$ through the peritoneal surface is associated with metabolic acidosis and the hypercapnia $(6,7)$. According to the aforementioned data there are some changes secondary to the insufflated $\mathrm{CO}_{2}$ during laparoscopy which were shown in both peritoeneal biopsies and labaratory investigations. Again it is well known that several different laparoscopic entry and the insufflation techniques have been used, most of the surgeons prefer one of these tecjniques based on the patients status and also their experience. Most commonly used techniques are open, direct trocar and the Veress needle entry techniques. According to our experience time to obtain enough intrabdominal pressure during insufflation is shorter after a direct trocar entry due to higher diameter of trocar compared to the Veress needle. Based on the data indicating peritoneal and intraabdomianl changes after a $\mathrm{CO}_{2}$ insufflation, we hypothesize that these changes may affect gastrointestinal functions so we aimed to assess the gastrointestinal function after different entry techniques in laparoscopic surgeries. 


\section{Material and Method}

Totally 108 women who underwent laparoscopic surgery due to different indications were included in randomized clinical trial at Department of obstetrics and gynecology unit of Zeynep Kamil Women and Children's health training and research hospital between March 2015 to September 2015. Study protocol was approved by the Zeynep Kamil Women and Children's Health training and Research Hospital Ethics committe (Approval number: 43/2015) and signed informed consent was obtained from each participant. This study was submitted to Clinical trials system and the register number is NCT02543853. Subjects were randomly assigned to two groups (randomization.com). Group 1 underwent laparascopic surgery inwhich pneumoperitoneum was obtained by direct trocar entry $(n=72)$ while in Group 2 Veress needle was used to create pneumoperitoneum before trocar insersion $(n=36)$. In both groups, $\mathrm{CO}_{2}$ insufflation was used to obtain enough space to perform surgical manipulations. 2:1 randomization was due to the cost of disposible veress needle. Medical histories, body mass index, operation time, estimated blood loss and time to peritoneal entry, time to maximal peritoneal pressure were all evaluated for each participants. Time from operation to first bowel sound and first defecation were recorded to assess the effect of different insufflation techniques. Duration of operation, blood loss, pre and postoperative hemoglobin levels were all evaluated. Inclusion criteria were: Women underwent gynecologic operation and women who were eligible for laparoscopy while exclusion criteria were women with previous gastrointestinal intervention and women with previous abdominal surgery, history of drug consumption, especially opioids, water and electrolyte disturbances, pancreatitis or peritonitis, no willingness to cooperate, intra- and post-operative complications, hypothyroidism, and muscular and neurological disorders. Body mass index was calculated by the formula $\mathrm{kg} / \mathrm{m}^{2}$. Indications for the sugery were classified as surgery for adnexal mass, leiomyoma, abnormal uterine bleeding or genital cancer. Duration of operation was determined by stop watch as minutes from entry to abdominal cavity to the end of surgery. Maximal abdominal pressure was accepted to be $16 \mathrm{~mm} \mathrm{Hg}$. Blood loss was calculated by the volume in reservoir of surgical aspirator. Data was collected by means of the interview form, questionnaires, and the physical examination of subjects. All patients were requested to record the time to the first bowel movement, passage of flatus, defecation. An independent medical staff who was unaware of the study protocol, visited the patients for every hour, and recorded the time of the first bowel flatulence movement, passage of flatus, defecation. Bowel sound was assessed from each abdominal quadrant at least 1 min duration by stethoscope. Bowel sound was considered to be positive if there was sound from at least one quadrant. Duration from operation to first flatus and defecation was recorded according to the patients declarations. All participants were mobilized 6 hour after operations. Again each participant underwent visual anologue scale questionnaire at postperative $6^{\text {th }}$ hour.

\section{Statistical analyses}

Data was analyzed using SPSS 15.0 for Windows. Pearson's correlation analysis or Spearman's correlation analysis was performed to assess the correlation between different variables Student $t$ test was used to compare continuous variables between the groups. $\mathrm{P}$ value $<0.05$ was accepted to be statistically significant.

\section{Results}

Mean age of the study population was 41.4 (19-75 years). Duration of operations ranged between 15 to 300 minutes. Minimum time to first flatulence was 6 while maximum duration was 50 hours. Correlation analyses showed a significant association between the technique for abdominal entry and postoperative hemoglobin $(\mathrm{r}=-0.253, \mathrm{p}=0.024)$ and hematocrit concentration $(\mathrm{r}=-0.272, \mathrm{p}=0.015)$, time to maximal intraabdominal pressure $(\mathrm{r}=0.3, \mathrm{p}=0.003)$ and the body mass index $(\mathrm{r}=-0.211, \mathrm{p}=0.004)$. Comparison of groups with different laparoscopic entry techniques showed a significant difference between groups in terms of postoperative hemoglobin and hematocrit concentrations, time to obtain maximal intraabdominal pressure and time to first flatulence $(\mathrm{p}<0.05$, Table 1$)$. No intra or postoperative complications was observed and there was no ileus or subileus case in groups.

Table 1: Comparison of groups with different laparoscopic entry techniques showed a significant difference between groups in terms of postoperative hemoglobin and hematocrit concentrations, time to obtain maximal intraabdominal pressure and time to first

\begin{tabular}{lllr}
\hline & $\begin{array}{c}\text { Veres (n=36) } \\
\text { Mean (SD) }\end{array}$ & $\begin{array}{l}\text { Trocar (n=72) } \\
\text { Mean (SD) }\end{array}$ & \\
\hline Age (years) & $39.45(13.7)$ & $42.3(10.6)$ & $\mathrm{NS}$ \\
Preop Hb (gr/dL) & $11.9(1.6)$ & $12.4(1.7)$ & $\mathrm{NS}$ \\
Preop Hct (\%) & $35.9(4.2)$ & $37.2(4.5)$ & $\mathrm{NS}$ \\
Postop Hgb (gr/dL) & $10.4(1.2)$ & $11.2(1.5)$ & $<0,05$ \\
Postop Hct (\%) & $31.7(3.1)$ & $33.9(4.4)$ & $<0,05$ \\
Postop vas score & $3.8(1.4)$ & $3.9(1.4)$ & $\mathrm{NS}$ \\
Time to max pressure (sec) & $68.6(37.1)$ & $45.6(15.1)$ & $<0,05$ \\
Gas volume (litre) & $3.06(1.5)$ & $3.65(1.1)$ & $\mathrm{NS}$ \\
Operation time (min) & $86.41(54.5)$ & $78.94(40.3)$ & $\mathrm{NS}$ \\
Estimated Blood loss (mL) & $149.06(177.8)$ & $140.95(168.4)$ & $\mathrm{NS}$ \\
Time to 1st bowel sound (hour) & $5.23(2.7)$ & $5.73(2.6)$ & $\mathrm{NS}$ \\
Time to first flatulence (hour) & $17.81(5.4)$ & $22.14(9.7)$ & $<0,05$ \\
Time to first defecation (hour) & $42.1(17.8)$ & $44.83(19.3)$ & $\mathrm{NS}$ \\
BMl & $28.3(7.3)$ & $30.2(6.3)$ & $\mathrm{NS}$ \\
\hline
\end{tabular}




\section{Discussion}

In this study we tried to assessed the effect of different insufflation techniques on postoperative gastrointestinal functions. Our data revealed that direct or Veress needle entry methods were both safe to create pneumoperitoneum with similar postoperative gastrointestinal functions except for earlier first flatulence in Veress needle group while direct trocar entry was found to be associated with favorable postoperative blood count and shorter duration to obtain enough intraperitoneal pressure.

In laparoscopic surgery, gas insufflation into the peritoneal cavity can be performed through a Veress needle or trocar. According to the result of Cochrane review, direct trocar entry technique was found to be superior in terms unsuccessful entries, extraperitoneal insufflation and omental injury compared to Veress needle (8). In addition to the above mentioned advantages, direct trocar entry provides faster insufflation with its wider diameter. $\mathrm{CO}_{2}$ has been used for pneumoperitoneum formation and has been found to be associated with some structural, metabolic and functional changes on the peritoneum (2). Based on these changes, some intercellular gap formations have been defined. Macrophage accumulation and fast insufflated cold $\mathrm{CO}_{2}$ gas may interfere with the peritoneal cellular functions (9). These macrophages were shown move into the intercellular spaces, cause of the migration is not certain whether it is due to direct pressure effect or peritoneal stretching (2). All these peritoneal changes with the insufflated gas have been shown by microscopy objectively (1). According to the literature, intraabdominal pressure $>12 \mathrm{~mm}$ $\mathrm{Hg}$ is considered to be abdominal hypertension (10). Again it has been shown that increased intraabdominal pressure as it is seen in laparoscopy is associated with the decrease in intestinal perfusion and even ischemia (11-17). Increased intraabdominal pressure was also found to decrease mesenteric blood supply (18). So it is expected to see an abnormality in gastrointestinal motility due to intestinal edema secondary to the hypoperfusion (19). There have been some adaptive and compansatory mechanisms introduced and it was found that these compansatory mechanisms needs at least 5 minutes (20). There are also some other confounders that may interfere with the gut functions, in our study population we did not find any difference between groups in terms of operation time, maximal intraabdominal pressure, amount of gas insufflated and the estimated blood loss. Only difference we found was the time to obtain the maximal intaabdominal pressure. So in this study we tried to assess the gut functions between groups to show whether fast insufflation interfere with the gut functions or not. We found that gut functions in groups with and without fast insufflation did not differ. This result showed that gastrointestinal system have enough compansatory potential to tolerate fast insufflation. The other difference was the postoperative complete blood counts which was favorable in group with direct trocar entry. Limitations of this study were small sample size and lack of data about histopathological changes resulted from insufflation.

In conclusion, in addition to the comparable safety issues and postoperative pain scores, direct trocar entry saves time, is associated with the favorable postoperative blood counts and according to our results faster insufflation can be well tolareted that direct and Veress needle entry methods had similar effect on postoperative gastrointestinal functions except for earlier first flatulence in group with Veress needle insufflation.

\section{References}

1. Schaeff B, Paolucci V, Henze A, Encke A. Electron microscopical changes to pneumoperitoneum after laparoscopic operations. Surg Endosc 1998;12:O36

2. Volz J, Koster S, Spacek Z, Paweletz N. Characteristic alterations of the peritoneum after carbon dioxide pneumoperitoneum. Surg Endosc 1999;13(6):611-614.

3. Heel KA, Hall JC. Peritoneal defenses and peritoneumassociated lymphoid tissue. Br J Surg 1996;83(8):10311036.

4. Hajri A, Mutter D, Wack S, Bastien C, Gury JF, Marescaux J et al. Dual effect of laparoscopy on cell-mediated immunity. Eur Surg Res 2000;32(5):261-266.

5. West MA, Baker J, Bellingham J. Kinetics of decreased LPS-stimulated cytokine release by macrophages exposed to CO2. J Surg Res 1996;63(1):269-274.

6. McDermott JP, Regan MC, Page R, Stokes MA, Barry K, Moriarty DC et al. Cardiorespiratory effects of laparoscopy with and without gas insufflation. Arch Surg 1995;130(9):984-988.

7. Neuberger TJ, Andrus CH, Wittgen CM, Wade TP, Kaminski DL. Prospective comparison of helium versus carbon dioxide pneumoperitoneum. Gastrointest Endosc 1996;43(1):38-41.

8. Ahmad G. O’Flynn H. Duffy JM. Phillips K. Watson A. Laparoscopic entry techniques. Cochrane Database Syst Rev 2012;2.

9. Puttick MI, Scott-Coombes DM, Dye J, Nduka CC, Menzies- Gow NM, Mansfield AO et al. Comparison of immunologic and physiologic effects of $\mathrm{CO}_{2}$ pneumoperitoneum at room and body temperatures. Surg Endosc 1999;13(6):572-575.

10. Kirkpatrick AW, Roberts DJ, De Waele J, Jaeschke R, Malbrain ML, et al. Intra-abdominal hypertension and the abdominal compartment syndrome: updated consensus definitions and clinical practice guidelines from the World Society of the Abdominal Compartment Syndrome. Intensive Care Med 2013;39(7):1190-1206.

11. Kotidis E, Papavramidis T, Ioannidis K, Koliakos G, Lazou T, et al. Can chronic intra-abdominal hypertension cause oxidative stress to the abdominal wall muscles? An experimental study. J Surg Res 2012;176(1):102-107. 
12. Gong G, Wang P, Ding W, Zhao Y, Li J. The role of oxygen-free radical in the apoptosis of enterocytes and bacterial translocation in abdominal compartment syndrome. Free Radic Res 2009;43(5):470-477.

13. Ihtiyar E. Yasar NF. Erkasap N. Koken T. Tosun M. et al. Effects of doxycycline on renal ischemia reperfusion injury induced by abdominal compartment syndrome. J Surg Res 2011;167(1):113-120.

14. Duzgun AP, Gulgez B, Ozmutlu A, Ertorul D, Bugdayci $\mathrm{G}$, et al. The relationship between intestinal hypoperfusion and serum d-lactate levels during experimental intra-abdominal hypertension. Dig Dis Sci 2006;51(12):24002403.

15. Moore-Olufemi SD, Xue H, Allen SJ, Moore FA, Stewart $\mathrm{RH}$, et al. Effects of primary and secondary intra-abdominal hypertension on mesenteric lymph flow: implications for the abdominal compartment syndrome. Shock 2005; 23(6):571-575.

16. Kaussen T, Srinivasan PK, Afify M, Herweg C, Tolba R, et al. Influence of two different levels of intra-abdominal hypertension on bacterial translocation in a porcine model. Ann Intensive Care 2012 Jul;2 Suppl 1:S17.

17. Cheng J, Wei Z, Liu X, Li X, Yuan Z, et al. The role of intestinal mucosa injury induced by intra-abdominal hypertension in the development of abdominal compartment syndrome and multiple organ dysfunction syndrome. Crit Care 2013 Dec 9;17(6):R283.

18. Diebel LN, Dulchavsky SA, Wilson RF. Effect of increased intra-abdominal pressure on mesenteric arterial and intestinal mucosal blood flow. J Trauma 1992 Jul;33(1):45-9.

19. Leng Y. Zhang K. Fan J. Min Y. Qinggang G. Le C. et al. "Effect of Acute, Slightly Increased Intra-Abdominal Pressure on Intestinal Permeability and Oxidative Stress in a Rat Model. PLoS One 2014;9(2):e115133

20. Kotzampassi K, Kapanidis N, Kazamias P, Eleftheriadis E. Hemodynamic events in the peritoneal environment during pneumoperitoneum in dogs. Surg Endosc 1993 Nov-Dec;7(6):494-9. 